TecnoLógicas

ISSN-p 0123-7799

ISSN-e 2256-5337

Vol. 22, No. 45, pp. 73-90

Mayo-agosto de 2019

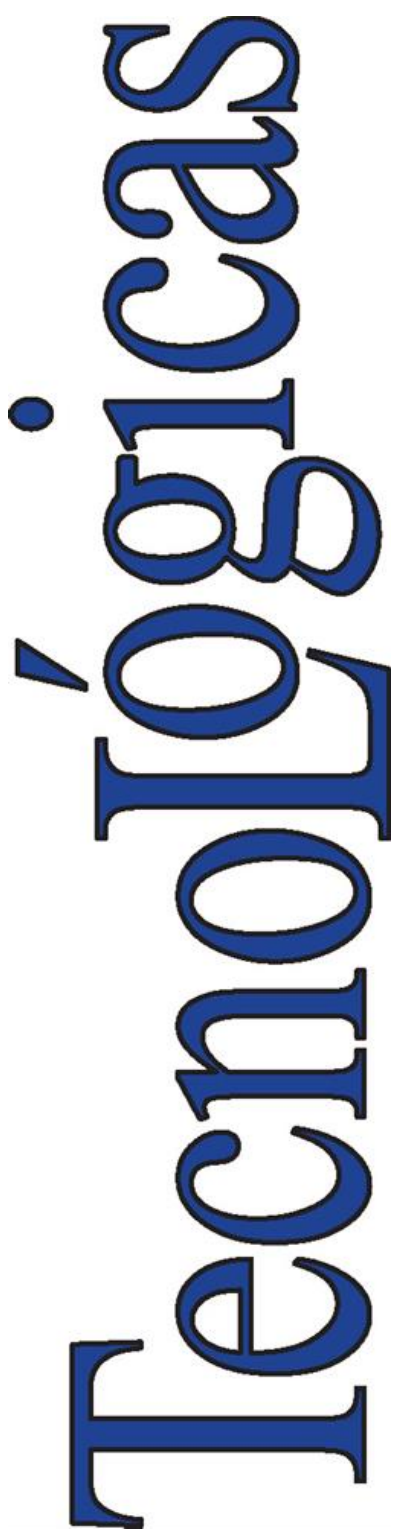

(c) Instituto Tecnológico Metropolitano Este trabajo está licenciado bajo una Licencia Internacional Creative Commons Atribución (CC BY-NC-SA)

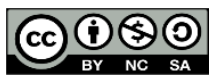

Artículo de Investigación/Research Article

\section{Desarrollo de compuestos XLPE con retardantes de llama de baja toxicidad para aislamiento de cables}

\section{Development of XLPE compounds with low- toxicity flame retardant for cable insulation}

\author{
Leonardo Gutiérrez $\mathbf{Q}^{1}$, y Jose H. Mina $\mathbb{D}^{2}$
}

Recibido: 26 de octubre de 2018

Aceptado: 25 de abril de 2019

\section{Cómo citar / How to cite}

L. Gutiérrez, J.H. Mina "Desarrollo de compuestos XLPE con retardantes de llama de baja toxicidad para aislamiento de cables". TecnoLógicas, vol. 22, no. 45, pp. 73-90, 2019. https://doi.org/10.22430/22565337.1104
1 Ingeniero de materiales, Grupo Materiales Compuestos, Universidad del Valle, Cali-Colombia, leonardo.gutierrez@correounivalle.edu.co

2 Ingeniero de materiales, Grupo Materiales Compuestos, Universidad del Valle, Cali-Colombia, jose.mina@correounivalle.edu.co 


\section{Resumen}

Se desarrollaron nueve formulaciones de un masterbatch retardante a la llama de bajo impacto medioambiental y se evaluó la influencia de su incorporación en un polietileno lineal de baja densidad, injertado con un agente de entrecruzamiento viniltrimetoxisilano, para su posterior reticulación con el fin de obtener un polietileno entrecruzado con retardancia a la llama. Los masterbatch retardantes a la llama se prepararon a partir del uso de N, N-etilen bis(tetrabromoftalamida), mezclado con: trióxido de antimonio, borato de zinc, polietileno lineal de baja densidad, agente de dispersión y cera polietilenica, variando el N,N-etilen bis(tetrabromoftalamida) (36, 42 y $45 \%$ ) y el agente de dispersión $(0.50,1.25$ y $2.00 \%$ ), mientras se mantuvo constante el resto de componentes. Posteriormente, los nueve masterbatch desarrollados se mezclaron con el polietileno lineal de baja densidad injertado para la producción de nueve compuestos de polietileno entrecruzado retardante de lama que luego se conformaron en forma de placas y aislamiento de cable 14 AWG para su caracterización fisicoquímica. Se encontró que el nivel empleado tanto en las cargas (N,Netilen bis-tetrabromoftalamida, trióxido de antimonio y borato de zinc) como en el agente de dispersión condicionaron el nivel de entrecruzamiento estimado a partir del contenido de gel, las características de flujo y el comportamiento frente a la llama del material; siendo favorecidas las primeras dos propiedades con el incremento del agente de dispersión, en tanto que la última se vio mejorada con el aumento en la proporción de las cargas usadas. De igual manera, la evaluación de un compuesto de polietileno entrecruzado retardante a la llama fabricado con un masterbatch retardante a la llama comercial, tomado como referencia, evidenció que algunas de las formulaciones trabajadas en esta investigación son susceptibles de ser empleadas para el aislamiento de cables eléctricos.

\section{Palabras clave}

Aislamiento de Cables, Polietileno Entrecruzado, Retardantes de Llama, Masterbatch.

\section{Abstract}

Nine formulations of a flame retardant masterbatch with low environmental impact were developed, and the influence of their incorporation into a linear low-density polyethylene, grafted with a vinyltrimethoxysilane crosslinking agent, was evaluated for further crosslinking in order to obtain a cross-linked polyethylene with flame retardancy. The MBFRs were prepared using N, N-ethylene bis (tetrabromophthalamide) blended with antimony trioxide, zinc borate, linear low-density polyethylene, dispersing agent, and polyethylene wax, varying the $\mathrm{N}$, N-ethylene bis (tetrabromophthalamide) (36, 42, and 45 $\%)$ and the dispersing agent $(0.50,1.25$, and $2.00 \%)$, while the rest of the components were kept constant. Subsequently, the nine developed flame retardant masterbatch were blended with the grafted linear low-density polyethylene to produce nine flame retardant crosslinked polyethylene compounds that were later shaped into plates and 14 AWG cable insulation for physicochemical characterization. The level used in both the fillers $(\mathrm{N}, \mathrm{N}$ ethylene bis-tetrabromophthalamide, antimony trioxide and zinc borate) and the dispersion agent conditioned the level of crosslinking estimated from the gel content, the flow performance, and the flame behavior of the material; the first two properties were favored by the increase in the dispersing agent, while the latter was improved with the increase in the proportion of fillers in the mixture. Likewise, the evaluation of the flame retardant cross-linked polyethylene compound manufactured with a commercial MBFRC, taken as a reference, showed that some of the formulations analyzed in this study can be used for electric cable insulation.

\section{Keywords}

Cable Insulation, Crosslinked Polyethylene, Flame Retardant, Masterbatch. 


\section{INTRODUCCIÓN}

El polietileno es un material termoplástico típico, lo cual limita su aplicación a temperaturas significativamente menores a su temperatura de fusión. Sin embargo, principalmente, a partir del polietileno de baja densidad lineal LLDPE se puede obtener el polietileno entrecruzado o reticulado (XLPE), que se caracteriza por ser un polímero termoestable con enlaces primarios entre cadenas vecinas, lo cual le confiere una mejor resistencia química $\mathrm{y}$ rigidez, así como mayor resistencia a la tracción y al cambio de temperatura; siendo utilizado entre otras aplicaciones para la protección y el aislamiento de líneas eléctricas de baja y media tensión[1]-[8]. Por otra parte, dada su naturaleza orgánica los polímeros son materiales muy sensibles al fuego, por lo cual para su uso en algunas aplicaciones requieren del mejoramiento de su respuesta a la llama mediante el uso de aditivos y concentrados retardantes de llama. Los primeros retardantes de llama empleados en materiales plásticos fueron compuestos orgánicos halogenados, con contenido de bromo y cloro principalmente, y compuestos organofosforados [9]. Desde 1960, los retardantes de llama bromados (BFR) se han utilizado en una amplia variedad de aplicaciones de uso generalizado en la sociedad moderna [10], como son el aislamiento de alambres y cables, debido a su bajo costo y alta eficiencia de retardancia a la llama [11], reduciendo la agresividad, la velocidad de propagación, la producción de humos y gases de combustión, minimizando así la pérdida de vidas y costos económicos asociados a la combustión de aislamientos de cables [12].

Algunos compuestos de boro, tales como los boratos de zinc (ZB), suelen utilizarse en combinación con el trióxido de antimonio (ATO) para mejorar la retardancia a la llama de los sistemas halogenados, actuando como supresores de humo, promoviendo la formación $\mathrm{y}$ estabilización de carbón [9], [11], [13].

La Unión Europea (UE) y el Gobierno de los Estados Unidos (US) han expresado su preocupación acerca del uso de los BFR. En este sentido, se han realizado esfuerzos para identificar y recoger información sobre los tipos de retardantes de llama aplicados en productos de consumo, para revisar y evaluar la toxicidad y el impacto ambiental de los principales compuestos actualmente usados. Existen numerosos estudios sobre este tipo de compuestos que indican persistencia y bioacumulación en el medio ambiente [14]-[22]. Por tal motivo, entidades como el Consejo de la Unión Europea y la Agencia de Protección Medioambiental de Estados Unidos (EPA), entre otras, han emitido reportes para restringir el uso de óxidos de difenilo bromados como retardantes de llama (Directiva RoHS), debido a que durante la combustión se pueden formar furanos y dioxinas bromadas altamente tóxicas y potencialmente cancerígenas [23]-[28].

Las agencias de Protección ambiental de Estados Unidos y Dinamarca concluyeron que el N,N-etilen bis (tetrabromoftalamida) (EBTPI) no tiene impactos negativos mayores sobre el medio ambiente, la salud y/o seguridad de los consumidores como el Decabromodifenil éter (Deca-BDE) [24], [29]. El EBTPI tiene una gama de aplicaciones similares a las del Deca-BDE y ha sido producido y comercializado como sustituto de uso general por las mismas empresas que producen Deca-BDE. Aunque es más costoso que el Deca-BDE, el EBTPI tiene propiedades técnicas superiores a las del Deca-BDE [24], [29]. De aquí que la tendencia actual es la de desarrollar y fabricar cables aislados que además de ser seguros, tengan el mínimo impacto posible sobre la salud y el medio ambiente. Resulta evidente entonces la necesidad de desarrollar compuestos poliméricos con sistemas retardantes de llama que 
cumplan con nuevas regulaciones, estándares y métodos de prueba.

De acuerdo con lo comentado, el propósito fundamental del trabajo de investigación fue desarrollar un aislamiento para cables basado en un polietileno reticulado y con retardancia a la llama XLPE-FR, a través de la incorporación de un masterbatch retardante a la llama (siglas en inglés MBFR) que contiene aditivos retardantes de llama de menor impacto medioambiental (EBTPI) con respecto a los compuestos restringidos basados en bromo (Deca-BDE), los cuales le confirieron al aislamiento de XLPE propiedades ignífugas con baja emisión de humos, conservando un desempeño adecuado para su aplicación en cables de bajo voltaje, según la norma UL 44.

\section{METODOLOGíA}

\subsection{Materiales}

En la fabricación del XLPE y como vehículo del MBFR se utilizó una resina base de polietileno lineal de baja densidad (LLDPE) de referencia CLNA 8400 con un índice de fluidez de $0.7 \mathrm{~g} / 10 \mathrm{~min}$, una densidad de $0.920 \mathrm{~g} / \mathrm{cm} 3$, una rigidez dieléctrica de $22 \mathrm{KV} / \mathrm{mm}$, una resistencia a la tensión de 17.65 MPa y una elongación a la rotura de $800 \%$, su presentación fue en gránulos y se adquirió en Hanwha. Con el fin de reticular el polietileno se empleó un agente viniltrimetoxisilano multicomponente de referencia Performix 1130 adquirido en Alchemix. Se empleó un concentrado de antioxidante y desactivador metálico marca Stab 10 de Alchemix. Para la preparación del concentrado retardante de llama (MBFR) se usó: N, N-Etilen bis(tetrabromoftalamida)

(EBTPI) referencia Broshield 67B de St. Louis Group, Borato de zinc (ZB) referencia SmokeShield ZB4 de St. Louis Group, Trióxido de antimonio (ATO) referencia
Firegard ATO adquirido en de St. Louis Group, Ayuda de dispersión (DA) referencia TR 121 de Struktol, Cera polietilénica (Cera PE) referencia CWO312 adquirido en Zell Chemie Int. Finalmente, una Ayuda de proceso a base de teflón, proporcionada por la compañía Clariant con la referencia Cesa Process MB 026.

\subsection{Preparación del concentrado retardante a la llama (MBFR)}

La obtención del MBFR, de acuerdo con las diferentes formulaciones planteadas en la Tabla 1, se llevó a cabo en dos etapas. En la primera se utilizó un mezclador planetario P600 Brabender a una velocidad de $140 \mathrm{rpm}$ y temperatura ambiente. Se mezcló primero el EBTPI junto con el ATO y ZB durante 3 minutos y luego se adicionaron el resto de componentes (LLDPE, DA y Cera PE), mezclando por 3 minutos más. Posteriormente, el MBFR en polvo pasó a la segunda etapa de compounding, donde fue mezclado intensivamente en un LabCompounder Brabender constituido por una extrusora de doble tornillo (twin screw extruder). A la salida (cabezal) se obtienen un par de "espaguetis" de material fundido (melt) que se pasan a través de una canaleta de enfriamiento por agua, seguido de un dispositivo granulador (peletizador) para obtener finalmente el producto en forma de gránulos (pellets).

\subsection{Preparación del polietileno injertado (Grafting)}

Para la fabricación del LLDPE injertado primero se efectuó la infusión del silano en el material, mediante una mezcla manual utilizando un recipiente plástico durante 10 minutos; posteriormente se dejó reposar la mezcla por 24 horas antes de llevar a cabo la reacción de grafting (injertado) en el Lab-Compounder 
Brabender. Esta reacción se lleva a cabo sin que se presente entrecruzamiento, tal como se ilustra en la Fig. 1. El injerto del LDPE se realizó en tandas de 300 gramos.

Tabla 1. Composición de los concentrados retardantes a la llama (MBFR). Fuente: autores.

\begin{tabular}{ccc}
\hline Formulación & $\begin{array}{c}\text { EBTPI } \\
\text { (\% en masa) }\end{array}$ & $\begin{array}{c}D A \\
\text { (\% en masa) }\end{array}$ \\
\hline MBFR1 & 36 & 0.50 \\
MBFR2 & & 1.25 \\
MBFR3 & & 2.00 \\
MBFR4 & 42 & 0.50 \\
MBFR5 & & 1.25 \\
MBFR6 & & 2.00 \\
MBFR7 & 45 & 0.50 \\
MBFR8 & & 1.25 \\
MBFR9 & & 2.00 \\
\hline
\end{tabular}

\subsection{Obtención del compuesto con retardante a la Ilama (XLPE-FR)}

Para la fabricación del aislamiento de polietileno XLPE-FR, se mezcla el LLDPE injertado con el Masterbatch retardante a la llama (MBFR), previamente preparado; además, se incorpora la ayuda de proceso Cesa Process MB 026. El compuesto XLPEFR consistió de una mezcla de $73 \%$ del LLDPE injertado, $25 \%$ de MBFR y $2 \%$ de ayuda de proceso. Para la posterior medición de las variables de respuesta los compuestos XLPE-FR, se sometieron a dos procesos de transformación diferentes, con el fin de obtener como producto terminado tanto placas como cable aislado con el compuesto XLPE-FR. La producción de cable se realizó por medio de extrusión de tornillo simple en un plastógrafo Brabender (relación L/D 25:1, relación de compresión de 3:1 y diámetro de $19 \mathrm{~mm}$ ). La extrusora se acopla a un dispositivo transportador (conveyor), que le suministra un conductor 14 AWG con ayuda de un carrete y simultáneamente el material fundido es depositado sobre el alambre de cobre al pasar a través del cabezal de extrusión para obtener el cable, el cual se enfría al pasar por una canal con agua a temperatura ambiente. La producción de placas se realizó mediante moldeo por compresión en caliente con ayuda de una prensa hidráulica de marca PHI, que fue trabajada a una temperatura de $160{ }^{\circ} \mathrm{C}$, una presión de 25000 PSI y un tiempo de 5 min. Posteriormente se apagó el mecanismo generador de calor y se abrió el sistema de enfriamiento, conservando la presión, a fin de retirar la placa cuando la temperatura había descendido lo suficiente para que el material se encontrara bien solidificado $\left(40-50{ }^{\circ} \mathrm{C}\right)$. De igual manera, con fines comparativos, se evaluó bajo las mismas condiciones un material de aislamiento "XLPE-FRC" elaborado con un MBFR comercial de referencia DFDB 5400 del proveedor DOW, empleando las mismas proporciones de componentes especificadas anteriormente $(73 \%$ de XLPE, $25 \%$ de MBFR y $2 \%$ ).

\subsection{Entrecruzamiento del compuesto XLPE- FR}

Para efectos de este estudio, se aceleró el proceso de reticulación o entrecruzamiento del XLPE (provenientes de placas y cable), mediante su inmersión en agua a $90{ }^{\circ} \mathrm{C}$ durante 48 horas, promoviendo la reacción de entrecruzamiento especificada en la Fig. 2.

\subsection{Determinación del contenido de gel (ASTM D 2765)}

El contenido de gel o fracción insoluble de los compuestos XLPE-FR se determinó de acuerdo con la norma ASTM D2765 método A [30]. Una muestra de aislamiento XLPE-FR de aproximadamente $0.3 \mathrm{~g}$ se envolvió en una jaula hecha con malla (mesh 120) de acero inoxidable y la extracción se hizo con decahidronaftaleno con $1 \quad \%$ de antioxidante (Irganox 1010, Ciba-Geigy) durante 6 horas. Posterior a la extracción, 
las muestras se pesaron antes y después de ser secadas en un horno, provisto de un sistema de vacío a $55{ }^{\circ} \mathrm{C}$, hasta que la masa fue constante [31]-[34]. El contenido de gel y el porcentaje de extracción fueron calculados utilizando (1) y (2), respectivamente.

Cont.Gel $(\%)=100-$ Extracción $(\%)$

Extracción $(\%)=\left[\frac{\left(\mathrm{W}_{3}-\mathrm{W}_{4}\right)}{(1-\mathrm{F})\left(\mathrm{W}_{2}-\mathrm{W}_{1}\right)}\right] \times 100$

(1)<smiles>CCCC(CCC)C[Si](OC)(OC)OC</smiles>

(2)
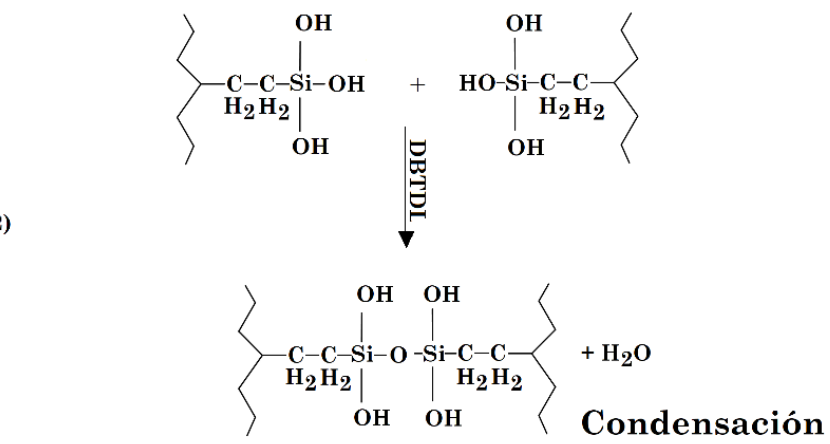

Fig. 1. Reacción de injerto (grafting) del silano en el LLDPE. Fuente: Autores

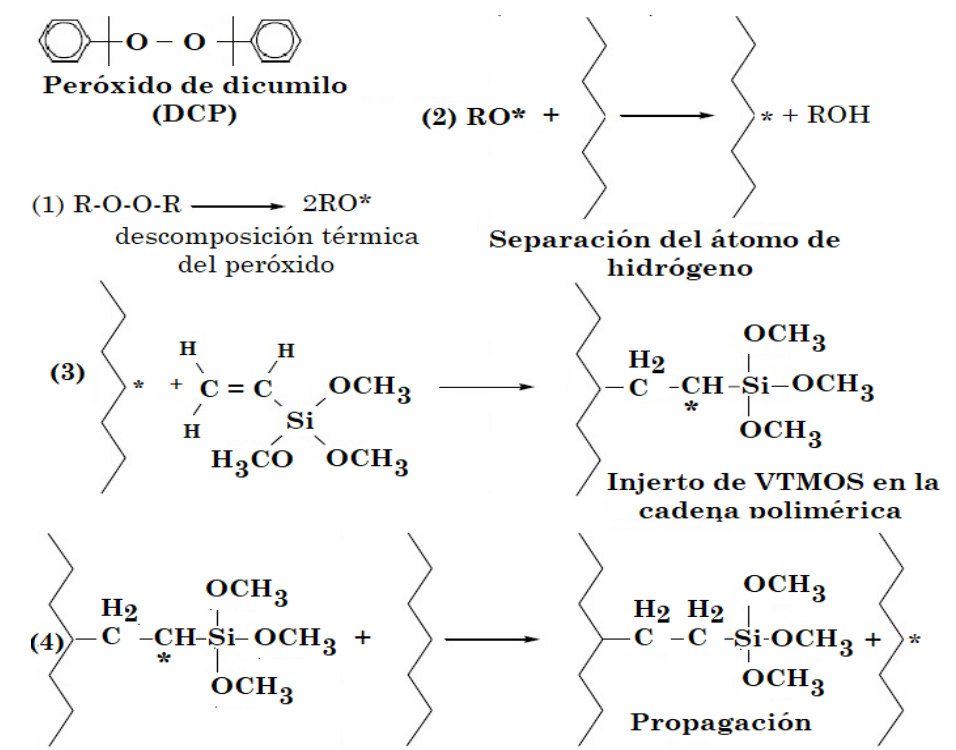

Fig.2. Reacción de entrecruzamiento del XLPE. Fuente: autores

\section{Hidrólisis}

dos, un lado abierto)

$\mathrm{W}_{2}=$ Masa de la muestra y la bolsa (sellada en tres lados, un lado abierto)

$\mathrm{W}_{3}=$ Masa de la muestra y la jaula, después de haber sido cerrada con grapas

$\mathrm{W}_{4}=$ Masa de la muestra y la jaula después de la extracción y secado

$\mathrm{F}=$ Fracción de relleno (que debe ser insoluble en decahidronaftaleno) en el compuesto de polietileno

[78] TecnoLógicas, ISSN-p 0123-7799 / ISSN-e 2256-5337, Vol. 22, No. 45, mayo-agosto de 2019, pp. 73-90 


\subsection{Determinación del Índice de Oxígeno (LOI) (ASTM D 2863)}

La determinación del índice de oxígeno de los compuestos XLPE-FR, se llevó a cabo con el propósito de caracterizar el comportamiento de los aislamientos frente a la combustión y fue efectuada teniendo en cuenta el método de ensayo descrito en la norma ASTM D 2863 [35], considerando como especímenes de prueba piezas rectangulares de $13 \times 4 \mathrm{~cm}$ moldeados por compresión, los cuales fueron quemados con un flujo constante $(\sim 20 \mathrm{~L} / \mathrm{min})$ de una mezcla entre oxígeno y nitrógeno. El conteniendo inicial de oxígeno fue un $50 \%$, y se comenzó a disminuir gradualmente su proporción, manteniendo constante el flujo de la mezcla de gases. La lectura que registró el equipo cuando se logró extinguir la llama, es la concentración mínima de oxígeno necesaria para mantener la combustión de la muestra.

\subsection{Realización de prueba de Llama Horizontal (UL 44)}

Una muestra de cable de $30 \mathrm{~cm}$ fue sometida a una llama de $500 \mathrm{~W}$, aplicada por un quemador en su punto medio por un periodo de $30 \mathrm{~s}$. El requisito de la norma UL 44 establece que la longitud quemada en el aislamiento del cable, después de la aplicación de la llama, no debe sobrepasar los $10 \mathrm{~cm}$ medidos simétricamente con respecto al punto de aplicación $(5 \mathrm{~cm}$ a cada lado), y no debe haber presencia de partículas o gotas emitidas durante 0 después de la exposición a la llama, evitando encender un tendido de algodón dispuesto en el piso del compartimento [36].

\subsection{Caracterización reométrica}

La presión generada por el material en la extrusora, la cantidad de material a la salida del cabezal de extrusión por fracción de tiempo y el torque de extrusión, fueron los parámetros medidos durante el proceso de extrusión del material de aislamiento XLPE-FR. La medición de estas variables fue realizada a diferentes velocidades del tornillo durante el proceso de extrusión (1/min o RPM). El análisis de los resultados se enfoca en el efecto que tienen en el XLPE-FR el contenido de aditivo antillama EBTPI/ATO/ZB (carga) y/o del agente de dispersión empleados para cada MBFR.

\subsection{Resistencia de aislamiento (UL 44)}

Empleando un megóhmetro se realizó la medición de resistencia eléctrica del aislamiento, aplicando un voltaje DC de $500 \mathrm{~V}$ durante 1 minuto, a una muestra de cable de al menos 30 metros de longitud sumergida en un tanque de agua a $15^{\circ} \mathrm{C}$. Dado que la medición normalmente se realiza en agua a temperatura ambiente $\left(\sim 25^{\circ} \mathrm{C}\right)$, se debió entonces aplicar en el cálculo del valor de resistencia de aislamiento, el correspondiente factor de corrección por temperatura de acuerdo con (3).

$R A=f R L$

Dónde:

RA: Resistencia de aislamiento (GQ.m) f: Factor de corrección por temperatura $\mathrm{R}$ : Lectura de resistencia eléctrica (GS) L: Longitud de la muestra de cable (m)

\subsection{Porcentaje de reticulación (Hot Creep y Hot Set) (UL 44)}

En esta prueba se midió la eficacia de la reticulación del aislamiento por medio de la determinación del alargamiento sufrido por el material aislante a una temperatura de $150^{\circ} \mathrm{C}$, bajo la acción de una carga equivalente a 20.4 veces la sección transversal del aislamiento y su posterior recuperación después de la eliminación de la carga. 
Se tomó una muestra de tres probetas extraídas del aislamiento del cable a ensayar. Las probetas fueron tubulares. Se marcó los $25 \mathrm{~mm}$ centrales de la probeta y se le colocó la mordaza superior. En el extremo inferior se le aplicó el peso prescrito para ejercer la fuerza de tracción definida para el material. El peso corresponde a 20.4 veces el área transversal del aislamiento.

Una vez el horno alcanzó la temperatura de ensayo, se suspendieron las probetas con el peso, en el interior del mismo durante el tiempo prescrito (en el caso del XLPE son 15 minutos a $150^{\circ} \mathrm{C}$ ). Pasado este tiempo se midió la distancia entre los trazos para calcular el alargamiento. A continuación, se cortó la probeta a ras de la mordaza para suprimir la fuerza de tracción y se dejó reposar durante 5 minutos en el interior del horno. Se retiró la probeta del horno y se esperó a que se enfriara a temperatura ambiente (5 a 10 minutos.). Pasado este tiempo, se volvió a medir la distancia entre los trazos, en frío y sin peso.

\section{RESULTADOS Y DISCUSIÓN}

\subsection{Contenido de Gel (ASTM D 2765)}

El grado de reticulación de los aislamientos XLPE-FR fue determinado a partir de la medición del contenido de gel de los especímenes de prueba resultantes del proceso de extrusión del polietileno injertado mezclado con cada uno de los nueve compuestos retardantes a la llama MBFR especificados previamente en la Tabla 1. De igual manera, se estudió el aislamiento XLPE-FRC que cuenta con el MBFR comercial. En la Fig. 3 se muestra la relación existente entre el contenido de gel del XLPE-FR, con su contenido de cargas y el contenido de agente de dispersión empleado para la fabricación del MBFR Todos los compuestos XLPE trabajados superan un contenido de gel del $52 \%$. En este sentido, es importante comentar que se ha encontrado que aislamientos con contenidos de gel superiores al $50 \%$ presentan un nivel de entrecruzamiento adecuado para su aplicación en cables eléctricos [37]-[39].

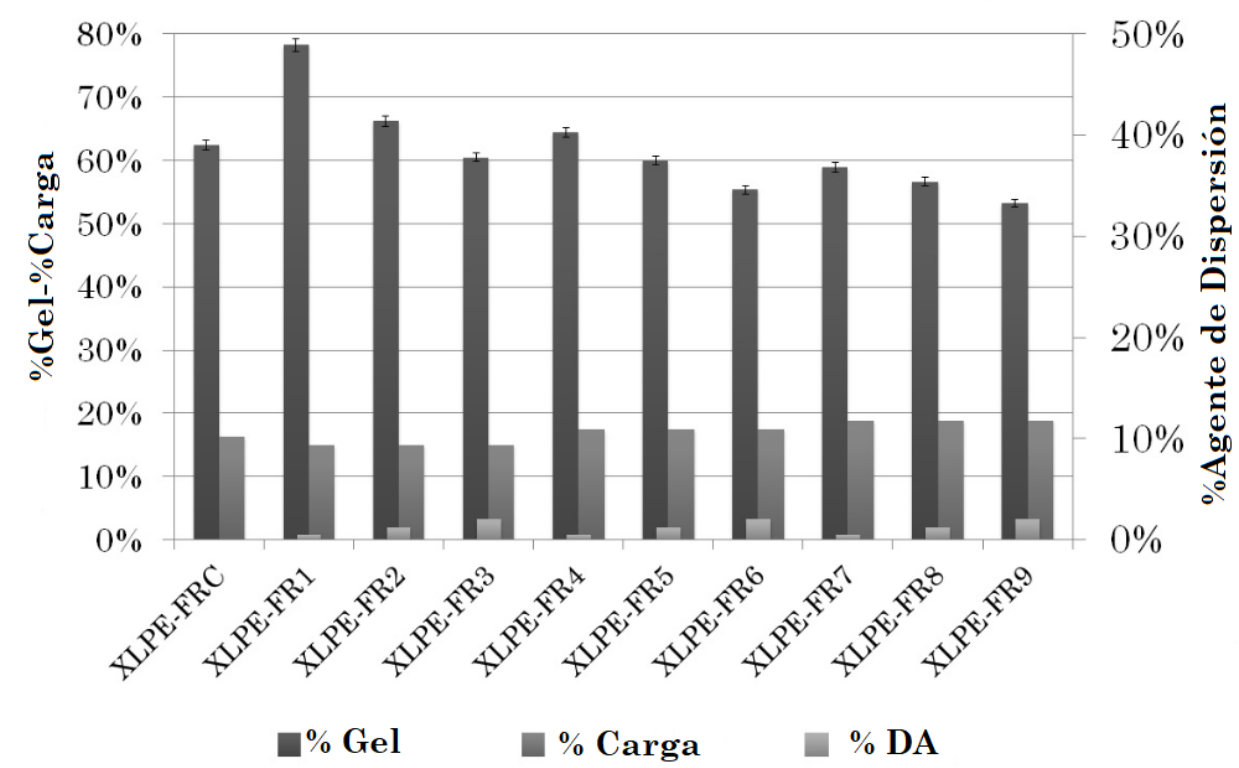

Fig. 3. Contenido de gel, de carga y agente de dispersión del MBFR en los XLPE-FR. Fuente: autores 
Para los XLPE-FR que mantienen constante su nivel de carga, se evidencia que, al aumentar el contenido de agente de dispersión en el MBFR, el valor del contenido de gel disminuye. Es el caso de los XLPE-FR1, XLPE-FR2 y XLPE-FR3; los cuales son fabricados con MBFR que presentan un mismo nivel de cargas, pero variaciones en el contenido del agente de dispersión. Siendo este valor bajo en el MBFR1, medio en el MBFR2 y alto en el MBFR3. Entre estos tres aislamientos, el contenido de gel fue mayor para el material que contiene el MBFR1 y menor para el que contiene el MBFR3, dejando con un valor intermedio al que contiene el MBFR2.

$\mathrm{Al}$ observar los aislamientos que son fabricados con los MBFR que presentaban un mismo contenido de agente de dispersión, pero niveles de carga diferentes, se encuentra que entre mayor sea el contenido de carga del MBFR, menor es el contenido de gel del aislamiento. En este sentido, el entrecruzamiento del XLPE-FR, se favorece en los aislamientos que se caracterizan por ser fabricados con bajos niveles de cargas y bajos niveles de agente de dispersión. El aislamiento que presentó un mayor valor en el contenido de gel fue el XLPE-FR1, caracterizado por contar con el menor nivel de carga y agente de dispersión. Por el contrario, el aislamiento con menor valor de contenido de gel fue el aislamiento XLPE-FR9, que presentó el mayor contenido de cargas y de agente de dispersión.

El efecto que tienen las cargas de afectar el contenido de gel del aislamiento se debe, probablemente, a la reducción en la permeabilidad al agua que se da en la estructura del PE por un bloqueo físico que obstruye la difusión del agua al interior de esta. Adicionalmente, la superficie de las cargas puede absorber parcialmente el silano, reduciendo la cantidad disponible de este reactivo para que se den correctamente las reacciones que conducen a la reticulación del aislamiento [40], [41].
Azizi et al. [40] reportaron contenidos de gel entre 52 y $60 \%$ para compuestos XLPE-FR con ATH y $\mathrm{Sb}_{2} \mathrm{O}_{3}$, siendo similares a los resultados alcanzados en el presente estudio (53 a $78 \%$ ), para incorporaciones de cargas entre 7 y $23 \%$.

El agente de dispersión afecta la reticulación, debido a que es un componente que no presenta entrecruzamiento por interacción con el agente de reticulación, por tanto, su incorporación en el aislamiento involucra la reducción del contenido de resina para efectos del proceso de reticulación del aislamiento. Además, la oleamida que es el componente principal del agente de dispersión, al ser soluble solo en estado amorfo (mientras el aislamiento esta fundido), tiene el inconveniente que al solidificarse el LDPE migra hacia la superficie del material formando una capa lubricante que puede interferir bloqueando la permeabilidad del agua en la estructura del PE [42].

Por otra parte, es importante resaltar que tres de las formulaciones trabajadas (XLPE-FR1, XLPE-FR2 y XLPE-FR4) alcanzaron valores de contenido de gel superiores al obtenido con el del material con el XLPE-FRC (62\%) preparado con el MBFR comercial. No obstante, al desconocer la formulación de este compuesto, no es posible efectuar un mayor análisis.

\section{2 Índice de Oxígeno (LOI) (ASTM D 2863)}

El índice de oxígeno (LOI), fue medido en placas elaboradas con las diferentes formulaciones descritas en la Tabla 1, incluyendo adicionalmente la basada en el MBFR comercial (XLPE-FRC). Los valores de LOI se registran en la Tabla 2. Se encontró que, al aumentar el nivel de agente de dispersión, manteniendo constante el contenido de cargas, el valor de LOI se reduce ligeramente. 
Tabla 2. Índice de oxígeno de las diferentes formulaciones trabajadas. Fuente: autores.

\begin{tabular}{lc}
\hline Formulación & Índice de oxígeno (\%) \\
\hline XLPE-FR1 & 25 \\
XLPE-FR2 & 24 \\
XLPE-FR3 & 23 \\
XLPE-FR4 & 27 \\
XLPE-FR5 & 27 \\
XLPE-FR6 & 26 \\
XLPE-FR7 & 32 \\
XLPE-FR8 & 31 \\
XLPE-FR9 & 30 \\
XLPE-FRC & 27 \\
\hline
\end{tabular}

Para aislamientos fabricados con MBFRs de un mismo contenido de carga (como es el caso del MBFR1, MBFR2 y MBFR3), a medida que se aumenta el nivel de agente de dispersión en el MBFR, menor es el porcentaje de oxígeno necesario en el sistema para mantener la llama encendida, por tanto, el aislamiento es más combustible. El XLPE-FR3 presenta un menor LOI y resulta por consiguiente más combustible que el XLPE-FR 2 y el XLPE-FR1, respectivamente. Al mantener constante el contenido de agente de dispersión y aumentar el contenido de carga en el aislamiento, el valor de LOI se aumenta significativamente. En este sentido, el aislamiento XLP-FR1 que contiene un MBFR1 de menor nivel de cargas que el MBFR4 y el MBFR7 respectivamente, requiere de un menor porcentaje de oxígeno para mantener la combustión que los aislamientos XLPE-FR4 y XLPE-FR7.

De acuerdo con la patente presentada por Mahabir [43], los compuestos bromados con contenidos entre 19 y $23 \%$ de cargas FR, presentan valores de LOI que están en el intervalo de 28.4 a 30.9 \%. Los XLPE-FR fabricados $y$ evaluados en el presente estudio, poseen contenidos de carga retardante a la llama que están en el intervalo de 15 a 18,75\% con valores de LOI en el intervalo de 27 a $32 \%$. Dado que el típico valor de LOI del PE es $18 \%$ [44][45], y considerando que la concentración de oxígeno del aire es de aproximadamente $21 \%$ en volumen [46], es de esperar que la medición de LOI de un compuesto polimérico con retardante de llama supere ampliamente esta proporción [47]-[49].

\subsection{Prueba de llama horizontal (UL 44)}

Los datos obtenidos durante la evaluación de los aislamientos fabricados con los diferentes MBFR en la prueba de llama FT2 son reportados en la Tabla 3. Aquí se tuvo en consideración los parámetros definidos en la norma UL44, como son la presencia de partículas incandescentes y la longitud de cable afectada, además, como evidencia adicional, se registró el tiempo que el aislamiento requirió para extinguirse, de tal manera que se pudiera diferenciar el mejor desempeño ignifugo. A partir de los datos registrados en la Tabla 3, se puede observar que el contenido de cargas retardantes a la llama empleados en los compuestos MBFR1, MBFR2 y MBFR3 (60 $\%$ ), resultó no ser suficiente para dar cumplimiento a la prueba de llama en los aislamientos XLPE-FR 1, XLPE-FR2 y XLPE-FR3, debido a que la llama sobrepasó los límites establecidos por la norma y adicionalmente se genera desprendimiento de partículas incandescentes que queman el algodón ubicado en la base del montaje del experimento. Por otra parte, los resultados son satisfactorios en los aislamientos XLPE-FR4, XLPE-FR 5, XLPE-FR6, XLPE-FR 7, XLPE-FR8 y XLPE-FR9. En estos materiales la propagación de la llama fue simétrica y se mantuvo dentro de los límites indicados por la norma; así mismo, no se presentó goteo de material fundido, ni liberación de partículas incandescentes durante la prueba.

Con el fin de identificar el aislamiento con mejor comportamiento ignifugo, fue 
necesario evaluar el tiempo de extinción de la llama. Con base en ello, el mejor desempeño fue observado en la muestra XLPE-FR7 (tiempo de extinción $2.3 \mathrm{~s}$ ), la cual fue elaborada con el MBFR7, caracterizado por presentar un alto nivel de cargas (45\%) y menor contenido de ayuda de dispersión (0.5\%). Respecto a los aislamientos XLPE-FR6, XLPE-FR8 y XLPE-FR9, estos presentan un desempeño ignifugo similar al obtenido para el XLPEFRC al considerar el tiempo de extinción de la llama, la longitud afectada y el no desprendimiento de partículas que quemen el algodón.

\subsection{Caracterización reométrica}

La presión generada por el material en la extrusora, la cantidad de material a la salida del cabezal de extrusión por fracción de tiempo y el torque de extrusión, fueron los parámetros medidos durante el proceso de transformación del material de aislamiento XLPE-FR. La medición de estos parámetros fue realizada a diferentes velocidades del tornillo durante el proceso de extrusión (1/min o RPM). El análisis de los resultados se enfoca en el efecto que tienen en el XLPE-FR, el contenido de aditivo antillama EBTPI/ATO/ZB (carga) y/o el agente de dispersión empleados para cada MBFR. En la Fig. 4 se muestra la correspondencia existente entre la presión de extrusión y la velocidad del tornillo, para cada uno de los XLPE-FR evaluados. En ella se observa que a una velocidad de tornillo de 20 RPM los aislamientos procesados presentan presiones próximas entre sí. Sin embargo, a medida que la velocidad del tornillo aumenta, las presiones de los diferentes XLPE-FR difieren significativamente, lo que permite evidenciar el efecto que tienen las cargas y el agente de dispersión sobre el procesamiento del aislamiento.

En el caso de los aislamientos procesados con MBFR1, MBFR4 y MBFR7, para las diferentes velocidades evaluadas presentan los mayores valores de presión. Estos MBFR son los que contienen la menor proporción del agente de dispersión $(0.5 \%)$. Este aditivo al favorecer la dispersión de los ingredientes activos en los compuestos facilita el procesamiento del material, lo cual fue confirmado al

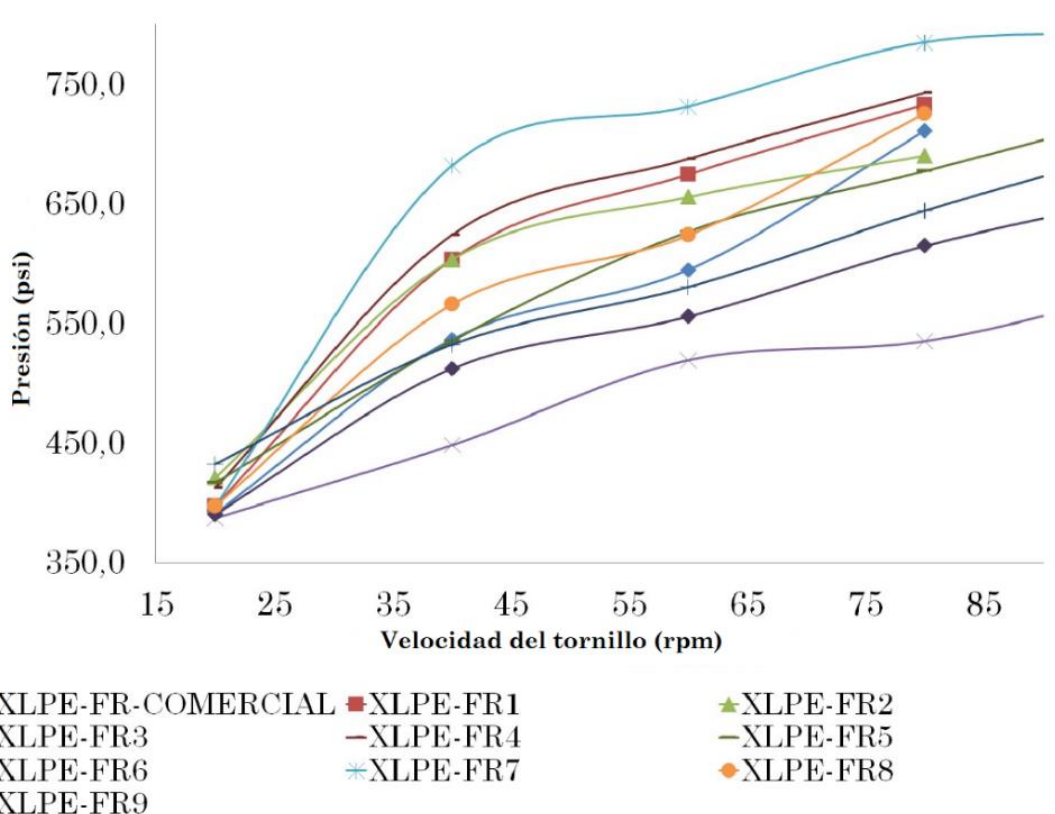

Fig. 4. Correspondencia existente entre la presión de extrusión y la velocidad del tornillo para los XLPE-FR. Fuente: autores 
Desarrollo de compuestos XLPE con retardantes de llama de baja toxicidad para aislamiento de cables

Tabla 3. Resultados Prueba de Llama Horizontal. Fuente: autores

\begin{tabular}{|c|c|c|c|c|}
\hline \multirow[t]{2}{*}{ Fórmula } & \multirow[t]{2}{*}{ Incandescentes } & $\begin{array}{l}\text { Longitud Afectada } \\
(\mathrm{mm})\end{array}$ & $\begin{array}{c}\text { Tiempo } \\
\text { extinción (s) }\end{array}$ & \multirow[t]{2}{*}{ Cumple } \\
\hline & & Promedio & Promedio & \\
\hline \multirow{3}{*}{ XLPE-FR1 } & si & & & no \\
\hline & si & $>100$ & 57.0 & no \\
\hline & si & & & no \\
\hline \multirow{3}{*}{ XLPE-FR2 } & si & & & no \\
\hline & si & $>100$ & 49.0 & no \\
\hline & si & & & no \\
\hline \multirow{3}{*}{ XLPE-FR3 } & si & & & no \\
\hline & si & $>100$ & 48.0 & no \\
\hline & si & & & no \\
\hline \multirow{3}{*}{ XLPE-FR4 } & no & & & si \\
\hline & no & 88.3 & 24.0 & si \\
\hline & no & & & si \\
\hline \multirow{3}{*}{ XLPE-FR5 } & no & & & si \\
\hline & no & 95.0 & 32.0 & si \\
\hline & no & & & si \\
\hline \multirow{3}{*}{ XLPE-FR6 } & no & & & si \\
\hline & no & 93.7 & 33.0 & si \\
\hline & no & & & si \\
\hline \multirow{3}{*}{ XLPE-FR7 } & no & & & si \\
\hline & no & 40.7 & 2.3 & si \\
\hline & no & & & si \\
\hline \multirow{3}{*}{ XLPE-FR8 } & no & & & si \\
\hline & no & 42.7 & 6.0 & si \\
\hline & no & & & si \\
\hline \multirow{3}{*}{ XLPE-FR9 } & no & & & si \\
\hline & no & 46.7 & 7.3 & si \\
\hline & no & & & si \\
\hline \multirow{3}{*}{ XLPE-FRC } & no & & & si \\
\hline & no & 39.7 & 6.7 & si \\
\hline & no & & & si \\
\hline
\end{tabular}

observar el comportamiento de los aislamientos procesados con los MBFR3, MBFR 6 y MBFR 9, los cuales presentan las menores presiones para las velocidades de tornillo evaluadas, gracias a su mayor contenido de agente de dispersión.

Referente al efecto que tienen las cargas sobre el procesamiento del aislamiento, como era de esperarse, cuando se procesan XLPE-FR empleando los MBFR con el mayor contenido de cargas, se generan mayores presiones en la extrusora para las diferentes velocidades evaluadas. La anterior afirmación es válida solo al comparar los MBFR con un mismo contenido de dosificación del agente de dispersión; es por esto que las presiones para las diferentes velocidades evaluadas del asilamiento procesado con el MBFR3 son inferiores a las del aislamiento procesado con el MBFR6 y este a su vez presenta menores presiones que el aislamiento fabricado con el MBFR9.

El efecto del nivel de carga sobre la presión de extrusión evidenciado en el presente análisis es similar al reportado en [46], quien encontró que en los aislamientos de XLPE-FR elaborados con Hidróxido de Aluminio (ATH), la dureza y viscosidad de las diversas formulaciones aumentaron con cada incremento de ATH, al seguir los valores registrados por las variables de respuesta (presión y torque) para un mismo perfil de extrusión. Los compuestos XLPE-FR a base de ATH, requieren de mayores niveles de 
dosificación para obtener resultados similares a los generados por los retardantes de llama bromados [50]-[52], es por esto que los valores de presión reportados por Marín [46], que están en el intervalo de 120 a 270 bares (1740 a 3915 PSI), son superiores a los valores obtenidos en el presente estudio (390 a 800 PSI).

El XLPE-FRC, fabricado con el MBFR comercial, tiene valores de presión, durante la extrusión, que son considerados intermedios con respecto a los nueve aislamientos resultantes del diseño de experimentos. Los XLPE-FR que más se acercan al comportamiento del XLPE-FRC son el XLPE-FR6, XLPE-FR5, XLPE-FR8 y XLPE-FR9. Para un proceso industrial resulta favorable que la presión de extrusión del aislamiento XLPE-FR no sea elevada, para ello se toma como patrón de referencia el XLPE-FRC, lo cual permitió identificar que los aislamientos con mejor comportamiento en un proceso industrial desde el punto de vista de la presión de extrusión, serían los aislamientos con valores de presión próximos e inferiores a los presentados por el XLPE-FRC.

En la Fig. 5 se presenta la correspondencia existente entre el flujo de salida del material en el cabezal de extrusión y la velocidad del tornillo. En ella se observa que el aislamiento XLPEFRC es el material con menores valores de flujo a la salida del cabezal de extrusión, para las diferentes velocidades evaluadas, cuando fue comparado con el flujo de salida de los nueve XLPE-FR experimentales. Para los aislamientos fabricados con los MBFR caracterizados por contener niveles de agente de dispersión bajo y medio, se puede observar que a medida que se incrementa la proporción de la carga sin modificar el nivel de agente de dispersión, el flujo de la mezcla XLPE-FR a la salida del cabezal tiende a reducirse. Este fenómeno no fue observado para los aislamientos fabricados con MBFR de alto nivel de agente dispersión, encontrándose adicionalmente que en estos a medida que se aumenta el nivel de carga en el MBFR, manteniendo constante el nivel de agente de dispersión, el flujo del XLPE-FR permanece estable. También es de resaltar que para aislamientos fabricados con los MBFR donde se mantiene constante el nivel de carga, al incrementar el contenido de agente de dispersión en el MBFR, el flujo del XLPE-FR a la salida del cabezal se incrementa. Lo anterior se observa para los niveles de carga bajo, medio y alto.

En la Fig. 6 se muestra la correspondencia existente entre el torque de extrusión y la velocidad del tornillo. En ella se observa que para las diferentes velocidades de tornillo evaluadas, los XLPE-FR fabricados con MBFR de bajo nivel de carga, presentan los menores valores de torque durante su extrusión. Además de ello, al aumentar el contenido de agente de dispersión en el MBFR manteniendo constante el contenido de carga, se observa que el valor del torque de extrusión del XLPE-FR se reduce. Este comportamiento se presenta para los XLPE-FR fabricados con medio y alto nivel de cargas. Para aislamientos XLPE-FR fabricados con MBFR del mismo contenido de agente de dispersión, pero con valores de carga variables, se encuentra que a mayor contenido de carga, el torque de extrusión del XLPE-FR aumenta. Adicionalmente, en la Fig. 6 se observa que el XLPE-FR3, es el aislamiento que presenta menores valores de torque durante su extrusión, para las diferentes velocidades de tornillo. El MBFR3 empleado para fabricar el XLPE-FR3, se caracteriza por contener el nivel de carga menor y el nivel de agente de dispersión mayor, reuniendo las dos condiciones requeridas para favorecer que se presente el menor torque de extrusión. El aislamiento XLPE-FR7, fabricado con el MBFR7, es el aislamiento que durante su extrusión requiere de mayor torque para ser procesado, debido a que el MBFR7 contiene el nivel de carga mayor y el nivel de agente de dispersión menor. 


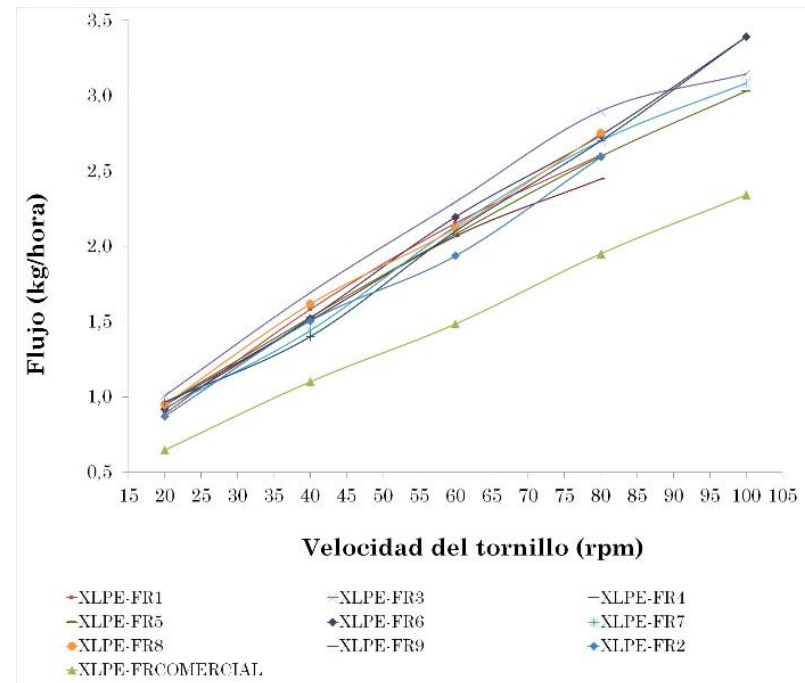

Fig. 5. Correspondencia existente entre el flujo de salida del XLPE-FR y la velocidad del tornillo. Fuente: autores.

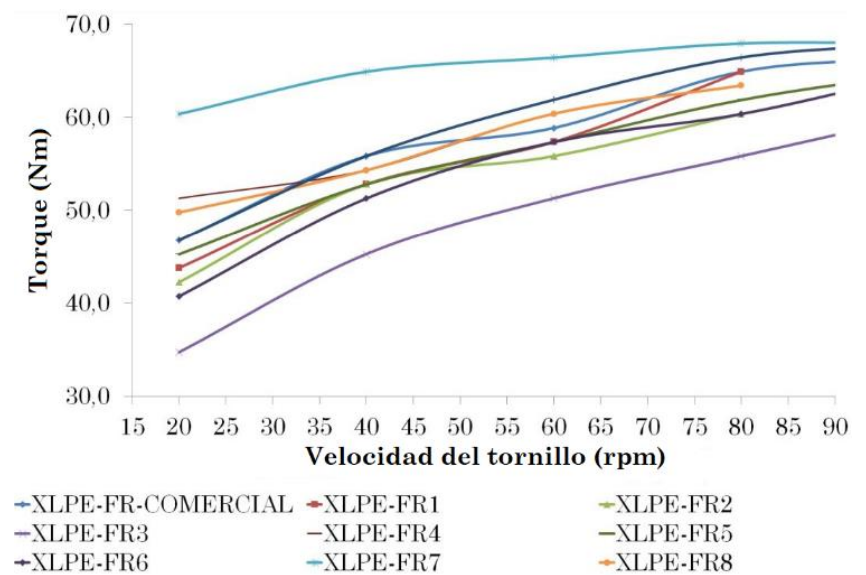

Fig. 6. Correspondencia existente entre el torque de extrusión del XLPE-FR y la velocidad del tornillo. Fuente: autores.

\subsection{Caracterización complementaria}

Con base en los resultados presentados y discutidos en las pruebas de contenido de gel, índice de oxígeno, llama horizontal y caracterización reométrica; el XLPE-FR de mejor desempeño corresponde al fabricado con el MBFR8.

La caracterización complementaria fue encaminada a evaluar el desempeño del aislamiento fabricado con el MBFR8, en las propiedades eléctricas y físico-térmicas contempladas en la norma UL 44.

En relación a la resistencia de aislamiento, fue determinada a partir de un cable 14 AWG fabricado con $25 \%$ de
MBFR8 de acuerdo con la metodología de obtención del compuesto con retardante a la llama (XLPE-FR), descrita en la sección 2.4. Igualmente, la resistencia de aislamiento de un cable aislado con XLPE sin retardante a la llama, fue medida para evaluar el efecto de la incorporación de cargas retardantes a la llama sobre las propiedades eléctricas del cable. En este sentido, el cable aislado con XLPE sin contenido de cargas presentó mejor resistencia de aislamiento (>72360 G $\Omega \mathrm{m})$ que la presentada por el cable aislado con

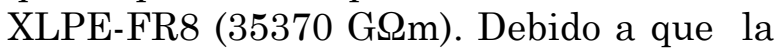
resistividad eléctrica de un material polimérico puede ser afectada 
negativamente cuando se incorporan compuestos iónicos como los aditivos ATO y ZB [53], resulta evidente que su influencia en el material representó la caída de la resistencia de aislamiento. No obstante, el requisito de la norma UL 44 (mínimo $810 \mathrm{G} \Omega \mathrm{m}$ ) fue ampliamente superado en el cable con aislamiento XLPE-FR8.

Por otro lado, los valores promedios obtenidos en la prueba de termofluencia para tres muestras de cada aislamiento son consignados en la Tabla 4. La prueba fue realizada sobre aislamientos de cables fabricados con el MBFR8.

Tabla 4. Termofluencia (hot creep elongation y hot creep set) de aislamientos XLPE y XLPEFR8. Fuente: Autores.

\begin{tabular}{lcc}
\hline Aislamiento & $\begin{array}{c}\text { Hot creep } \\
\text { (\%) }\end{array}$ & Hot Set (\%) \\
\hline XLPE & 21 & -4 \\
XLPE-FR8 & 34 & 2 \\
\hline
\end{tabular}

La norma UL 44 establece requisitos de Hot Creep y Hot Set máximos a cumplir, donde el aislamiento no debe exceder $100 \mathrm{y}$ $10 \%$ respectivamente, después de soportar un peso que equivale a 20.4 veces el área transversal del aislamiento. Los valores reportados en el presente estudio cumplieron satisfactoriamente con los requisitos establecidos por la norma UL 44 XLPE-FR, se evidencia que el XLPE presenta menor fluencia térmica bajo las condiciones establecidas por la prueba. Dicho comportamiento es atribuible a que el contenido de silano empleado para infusionar el aislamiento XLPE fue el mismo empleado para infusionar los aislamientos XLPE-FR, por tanto, es de esperar que el proceso de reticulación en el XLPE sea más efectivo, con una densidad de reticulación superior, ya que hay más silano disponible al no haber contenido de cargas que puedan interferir con el proceso de reticulación.
Algunas de las formulaciones de los masterbatch retardantes a la llama de bajo impacto medioambiental diseñados en el presente estudio, permitieron la fabricación de compuestos XLPE-FR que alcanzaron el cumplimiento de la prueba de llama FT2 especificada en la norma UL 44, conteniendo dentro de su formulación un componente bromado de baja persistencia y bioacumulación en el medio ambiente.

La variación de la proporción de las cargas (EBTI, ATO, ZB) y el agente de dispersión empleados en los masterbatch desarrollados, condicionaron el nivel de entrecruzamiento, la procesabilidad y el comportamiento frente a la llama de los compuestos XLPE-FR fabricados, de tal manera que niveles altos de carga promovieron un mejor comportamiento a la llama con menor procesabilidad mientras que las mayores proporciones del agente de dispersión propiciaron un comportamiento contrario.

Por su buen desempeño frente a las pruebas realizadas en la presente investigación, la formulación XLPE-FR8, fabricada con el MBFR8 de alto contenido de cargas y nivel medio de agente de dispersión, tiene factibilidad para ser usada en el aislamiento de cables eléctricos que requieran cumplir la norma UL 44. Dicho aislamiento presentó un desempeño reológico e ignifugo superior al XLPE-FR comercial y un grado de entrecruzamiento y desempeño eléctrico conveniente para la aplicación en aislamientos para cables.

\section{AGRADECIMIENTOS}

Los autores expresan su agradecimiento al Departamento de Investigación y Desarrollo de la empresa Cables para Energía y Telecomunicaciones CENTELSA.

\section{CONCLUSIONES}


Desarrollo de compuestos XLPE con retardantes de llama de baja toxicidad para aislamiento de cables

\section{REFERENCIAS}

[1] Y. Murata et al., "Development of High Voltage DC-XLPE Cable System," SEI Tech. Rev., no. 76, pp. 55-62, Apr 2013.

[2] N. Hampton, N. Rick Hartlein, H. Leenartsson, O. Harry Orton, and R. Ramachandran, "Long-Life XLPE Insulated Power Cable," Jicable 07, pp. 1-6, 2007.

[3] F. Ciuprina, G. Teissèdre, J. C. Filippini, A. Smedberg, A. Campus, and N. Hampton, "Chemical crosslinking of polyethylene and its effect on water tree initiation and propagation," IEEE Trans. Dielectr. Electr. Insul., vol. 17, no. 3, pp. 709-715,Jun. 2010. https://doi.org/10.1109/TDEI.2010.5492242.

[4] A. D. Quintero, "Propuesta de un portafolio de productos para el largo plazo en una compañia manufacturera de cables, utilizando mapas de ruta (Roadmaps)," Universidad del Valle, 2016.

[5] S. M. Tamboli, S. T. Mhaske, and D. D. Kale, "Crosslinked polyethylene," Indian J. Chem. Technol., vol. 11, pp. 853-864, Jul. 2004.

[6] V. a a Banks, P. H. Fraser, and a J. Willis, "Chapter 9 Wires and cables Principles of power cable design," in Newnes Electrical Power Engineer's Handbook, 2nd ed., D. Gracias and A. J. Willis, Eds. Oxford: Newnes, 2005, pp. 247-277.

[7] M. P. Ricardo Leon and A. M. Henao, "Dimensionamiento de cables de potencia aislados," Pontificia Bolivariana, 2013.

[8] A. Bhattacharya, J. W. Rawlins, and P. Ray, Polymer Grafting and Crosslinking. Hoboken, NJ, USA: John Wiley \& Sons, Inc., 2008. https://doi.org/10.1002/9780470414811.

[9] A. Wilkie, Charles; Morgan, Fire Retardancy of Polymeric Materials, 2nd ed. Boca Raton: CRC Press, Taylor \& Francis Group,Usa, 2010.

[10] S. Kemmlein, D. Herzke, and R. J. Law, "Brominated flame retardants in the European chemicals policy of REACHRegulation and determination in materials," J. Chromatogr. A, vol. 1216, no. 3, pp. 320333 , 2009 . https://doi.org/10.1016/j.chroma.2008.05.085.

[11] J. Markarian, "Regulatory issues drive developments in wire and cable," Plast. Addit. Compd., vol. 9, no. 4, pp. 32-35, Jul. $2007 . \quad$ https://doi.org/10.1016/S1464391X(07)70106-X.

[12] E. Díaz, R. Alonso, R. Valenciano, and I. M. De Arenaza, "Determinación de retardantes de llama bromados en plásticos de equipos eléctricos y electrónicos," Forum de Sostenibilidad, vol. 2, pp. 67-78,Jan. 2008.
[13] T. Akbulut, N. Ayrilmis, T. Dundar, and A. Durmus, "Effect of Boron and Phosphate Compounds on Thermal and Fire Properties of Wood / HDPE Composites," Int. J. Polym. Technol., vol. 3, no. 2, pp. 67-75,Aug. 2011.

[14] S. Gu et al., "Brominated flame retardants in marine environment focused on aquaculture area: Occurrence, source and bioaccumulation," Sci. Total Environ., vol. 601-602, pp. 1182-1191, Dec. 2017. https://doi.org/10.1016/j.scitotenv.2017.05.20 9.

[15] J. Guo, M. Venier, A. Salamova, and R. A. Hites, "Bioaccumulation of Dechloranes, organophosphate esters, and other flame retardants in Great Lakes fish," Sci. Total Environ., vol. 583, pp. 1-9, Apr. 2017. https://doi.org/10.1016/j.scitotenv.2016.11.06 3.

[16] M. Iqbal et al., "Legacy and emerging flame retardants (FRs) in the freshwater ecosystem: A review," Environ. Res., vol. 152, pp. 26-42, Jan. 2017. https://doi.org/10.1016/j.envres.2016.09.024.

[17] U. Kim, I. Lee, and J. Oh, "Occurrence, removal and release characteristics of dissolved brominated flame retardants and their potential metabolites in various kinds of wastewater," Environ. Pollut., vol. 218, pp. 551-557,nov. 2016. https://doi.org/10.1016/j.envpol.2016.07.037.

[18] J. P. Boon et al., "Levels of polybrominated diphenyl ether (PBDE) flame retardants in animals representing different trophic levels of the North Sea food Web.," Environ. Sci. Technol., vol. 36, no. 19, pp. 4025-4032, Oct. 2002. https://doi.org/10.1021/es0158298

[19] A. Covaci et al., "Hexabromocyclododecanes (HBCDs) in the Environment and Humans: A Review," Environ. Sci. Technol., vol. 40, no. 12, pp. 3679-3688, Jun. 2006. https://doi.org/10.1021/es0602492.

[20] R. J. Law et al., "Levels and trends of polybrominated diphenylethers and other brominated flame retardants in wildlife," Environ. Int., vol. 29, no. 6, pp. 757-770, Sep. $2003 . \quad$ https://doi.org/10.1016/S01604120(03)00110-7.

[21] K. Öberg, K. Warman, and T. Öberg, "Distribution and levels of brominated flame retardants in sewage sludge," Chemosphere, vol. 48, no. 8, pp. 805-809, Sep. 2002. https://doi.org/10.1016/S0045-6535(02)001133.

[22] A. Schecter, M. Pavuk, O. Päpke, J. J. Ryan, L. Birnbaum, and R. Rosen, "Polybrominated diphenyl ethers (PBDEs) in U.S. mothers' milk.," Environ. Health Perspect., vol. 111, no. 14, pp. 1723-1729, Nov. 2003. https://doi.org/10.1289/ehp.6466. 
Desarrollo de compuestos XLPE con retardantes de llama de baja toxicidad para aislamiento de cables

[23] Sub-Group on Review of the Priority Subtances List, "PolyBDEs EQS dossier 2011," 2011.

[24] U S Environmental Protection Agency (EPA), "An Alternatives Assessment for the Flame Retardant Decabromodiphenyl Ether (DecaBDE) Executive Summary," no. January. United States Environmental Protection Agency, p. (4)339--(4)359, 2014.

[25] U.S. Environmental Protection Agency (EPA), "Toxicological review of Decabromodiphenyl Ether (BDE-209)," United States Environmental Protection Agency, Washinton, DC, 2008.

[26] U. S. E. P. A. (EPA), "Toxicological review of 2,2',4,4',5-pentabromodiphenyl ether (BDE99)." Washington, pp. 1-74, 2008.

[27] United Nations (UNEP), "Report of the persistent organic pollutants review committee on the work of its seventh meeting," United Nations, Stockholm, 2011.

[28] Parlamento Europeo y del Consejo, Directiva 2002/95/CE del parlamento europeo y del consejo de 27 de enero de 2003 sobre restricciones a la utilización de determinadas sustancias peligrosas en aparatos eléctricos y electrónicos. Bruselas, 2003, pp. 19-23.

[29] F. Stuer-lauridsen, K. Cohr, and T. T. Andersen, "Health and Environmental Assessment of Alternatives to Deca-BDE in Electrical and Electronic Equipment," Danish Ministry of the Environment, 2007.

[30] ASTM International, "ASTM D 2765. Standard Test Methods for Determination of Gel Content and Swell Ratio of Crosslinked Ethylene Plastics," Annual Book of ASTM Standards. ASTM International, West Conshohocken, pp. 1-8, 2016. https://doi.org/10.1520/D2765-16.

[31] G. L. Oliveira and M. F. Costa, "Optimization of process conditions, characterization and mechanical properties of silane crosslinked high-density polyethylene," Mater. Sci. Eng. A, vol. 527, no. 18-19, pp. 4593-4599, Jul. 2010. https://doi.org/10.1016/j.msea.2010.03.102.

[32] K. Sirisinha, M. Boonkongkaew, and S. Kositchaiyong, "The effect of silane carriers on silane grafting of high-density polyethylene and properties of crosslinked products," Polym. Test., vol. 29, no. 8, pp. 958-965, Dec. 2010. https://doi.org/10.1016/j.polymertesting. 2010 . 08.004.

[33] R. P. De Melo, V. D. O. Aguiar, and M. D. F. V. Marques, "Silane Crosslinked Polyethylene from Different Commercial PE's: Influence of Comonomer, Catalyst Type and Evaluation of HLPB as Crosslinking Coagent," Mater. Res., vol. 18, no. 2, pp. 313-
319, Apr 2015. https://doi.org/10.1590/15161439.303214.

[34] K. Sirisinha and M. Boonkongkaew, "Improved silane grafting of high-density polyethylene in the melt by using a binary initiator and the properties of silanecrosslinked products," J. Polym. Res., vol. 20, no. 4, p. 120, Apr. 2013. https://doi.org/10.1007/s10965-013-0120-x.

[35] ASTM International, "ASTM D 2863 - 00 Standard Test Method for Measuring the Minimum Oxygen Concentration to Support Candle-Like Combustion of Plastics (Oxygen Index)," Annu. B. ASTM Stand., vol. 8, no. 1, pp. 1-15, 2017. https://doi.org/10.1520/D2863-00.

[36] U. L. Inc, "Thermoset-Insulated Wires and Cables," UL 44 Standar Saf., pp. 30-31, 2018.

[37] S. S. Sengupta, A. Ghosh-Dastidar, A. Flory, D. McDaniel, and P. Smith, "Evolution of Crosslinks During Moisture Cure of Ethylene-vinylalkoxysilane Copolymers in Power Cables," Int. Wire Cable Symp., vol. 57, pp. 146-151, 2008.

[38] G. B. Shah, M. Fuzail, and J. Anwar, "Aspects of the crosslinking of polyethylene with vinyl silane," J. Appl. Polym. Sci., vol. 92, no. 6, pp. 3796-3803, 2004. https://doi.org/10.1002/app.20381.

[39] A. Ghosh-dastidar, S. S. Sengupta, A. Flory, and J. M. Cogen, "Effect of Silane Grafting Level and Crosslink Structure on Properties of Moisture Crosslinkable Systems," in Dow Chemical Company, 2008, vol. 57, pp. 138145 .

[40] H. Azizi, J. Barzin, and J. Morshedian, "Silane crosslinking of polyethylene: The effects of EVA, ATH and $\mathrm{Sb2O} 3$ on properties of the production in continuous grafting of LDPE," Express Polym. Lett., vol. 1 , no. 6, pp. 378-384, May. 2007. https://doi.org/10.3144/expresspolymlett.2007 .53 .

[41] J. Morshedian and P. M. Hosseinpour, "Polyethylene Cross-linking by Two-step Silane Method: A Review," Iran. Polym. J., vol. 18, no. 2, pp. 103-128, 2009.

[42] A. Maltby and R. E. Marquis, "Slip Additives for Film Extrusion," J. Plast. Film Sheeting, vol. 14, no. 2, pp. 111-120, Apr. 1998. https://doi.org/10.1177/875608799801400204.

[43] C. M. Mahabir, "US5430091 Moisture crosslinkable flame retardant compositions for cable applications," 1995.

[44] N. Wang, D. Xiang, P. Mo, and Y. Lu, "Flame retardant low density polyethylene with aluminium hydroxide / commercial fire retardants FR01 synergistic system," $A d v$. Mater. Res., vol. 652-654, pp. 485-489, Jan. 
Desarrollo de compuestos XLPE con retardantes de llama de baja toxicidad para aislamiento de cables

2013.

https://doi.org/10.4028/www.scientific.net/A MR.652-654.485.

[45] M. Bar, R. Alagirusamy, and A. Das, "Flame retardant polymer composites," Fibers Polym., vol. 16, no. 4, pp. 705-717, Apr. 2015. https://doi.org/10.1007/s12221-0150705-6.

[46] Y. M. Tovar, "Desarrollo de un compuesto de polietileno reticulable con retardante de llama libre de halogeno y baja emision de humo como material de aislamiento en la fabricación de cables para baja tension en la empres Centelsa," Universidad del Valle, 2012.

[47] T. R. Hull, A. Witkowski, and L. Hollingbery, "Fire retardant action of mineral fillers," Polym. Degrad. Stab., vol. 96, no. 8, pp. 1462-1469, Aug. 2011. https://doi.org/10.1016/j.polymdegradstab.20 11.05.006.

[48] A. Dasari, Z. Yu, G. Cai, and Y. Mai, "Recent developments in the fire retardancy of polymeric materials," Prog. Polym. Sci., vol. 38, no. 9, pp. 1357-1387, Sep. 2013. https://doi.org/10.1016/j.progpolymsci.2013.0 6.006 .

[49] A. Ramani and A. E. Dahoe, "On the performance and mechanism of brominated and halogen free fl ame retardants in formulations of glass fi bre reinforced poly ( butylene terephthalate )," Polym. Degrad. Stab., vol. 104, pp. 71-86, Jun. 2014. https://doi.org/10.1016/j.polymdegradstab.20 14.03.021.

[50] S.-Y. Lu and I. Hamerton, "Recent developments in the chemistry of halogenfree flame retardant polymers," Prog. Polym. Sci., vol. 27, no. 8, pp. 1661-1712, Oct. 2002. https://doi.org/10.1016/S0079-6700(02)000187.

[51] F. Laoutid, L. Bonnaud, M. Alexandre, J.-M. Lopez-Cuesta, and P. Dubois, "New prospects in flame retardant polymer materials: From fundamentals to nanocomposites," Mater. Sci. Eng. $R$ Reports, vol. 63, no. 3, pp. 100125, Jan. 2009. https://doi.org/10.1016/j.mser.2008.09.002.

[52] L. Qiu, R. Xie, P. Ding, and B. Qu, "Preparation and characterization of $\mathrm{Mg}$ $(\mathrm{OH}) 2$ nanoparticles and flame-retardant property of its nanocomposites with EVA," Compos. Struct., vol. 62, no. 3-4, pp. 391395,Jan. 2003. https://doi.org/10.1016/j.compstruct.2003.09. 010.

[53] V. Y. Ushakov, "Electric Strength of Dielectric Materials in a Hostile Environment," in Insulation of High-Voltage Equipment, 2004th ed., Springer-Verlag, Ed. Berlin: Springer-Verlag Berlin and Heidelberg GmbH \& Co. KG, 2004, pp. 195263. 